\title{
Application of 2-D hydrodynamic mathematical model in the regulation project of secondary suspended river
}

\author{
"Zhao Zheng ${ }^{1, \mathrm{a}}$, Tian $\mathrm{Wu}^{1, \mathrm{~b}}$, Tianjiao Cui ${ }^{1, \mathrm{c}}$ \\ ${ }^{1}$ Henan Yellow River Reconnaissance, Design and Research Institute, ZhengZhou 450003, China \\ azzcollan@163.com, b43456047@qq.com, xiaocuictj@163.com
}

\begin{abstract}
Keywords: secondary suspended river, 2-D hydrodynamic mathematical model
Abstract. The secondary suspended river in the lower of Yellow River has been developing seriously, Possibility of floods running along the main dike toe in the parts of reach, even the "rolling flow" maybe appear, the security of dike has been threaten greatly. To solve the problem of the secondary suspended river, the key method is to reduce elevation difference between the main river channel and the toe of the main dike. The control measure is extracting the sands from main channel and beach to the "dike river", which means the toe of the dike, by adopting machines. In this paper, by using the 2-D hydrodynamic mathematical model to analyze the change of the flow velocity in the dike river after filling with various schemes, it is necessary for us to find a reasonable scheme to solve the problem of the secondary suspended river by utilizing the calculation results of the 2-D hydrodynamic mathematical model.
\end{abstract}

\section{Introduction}

Due to the characteristics of Yellow River with big sediment concentration, the lower of Yellow River has become a famous secondary suspended river in the world. To reduce the threaten of secondary suspended river, lighten the flood burden of the dike, and prevent life and property of people who live in the beach from submerging. The secondary suspended river is serious, especially prone to rolling river, so it is very necessary to take effective project measures to regulate the secondary suspended river. To solve the problem of the secondary suspended river, the key method is to reduce elevation difference between the main river channel and the toe of the main dike. The ordinary measure is extracting the sands from main channel and beach to the "dike river", which means the toe of the dike, by adopting machines.

The main building of the dike river filling project is the filling soil, which has three factors: length, width and height. The length of the filling soil equals the length of the dike, and the width is defined as the actual width of the dike river. It is very important to introduce the height of the filling soil, because the effectiveness of regulation project is ensure by it directly. In this paper, the author used the 2-D hydrodynamic mathematical model to decide the height of the filling soil.

\section{The Basic Principle and Mathematical Model}

The basic equations. Body fitted orthogonal curvilinear coordinate's model control two-dimensional mathematical equations, form as follows (Eq.1 3):

Equation of continuity:

$$
\frac{\partial h}{\partial t}+\frac{1}{C_{\varsigma} C_{\eta}}\left[\frac{\partial\left(C_{\eta} H u\right)}{\partial \varsigma}+\frac{\partial\left(C_{\varsigma} H u\right)}{\partial \eta}\right]=0
$$

The momentum equation:

$$
\begin{aligned}
& \frac{\partial(H u)}{\partial t}+\frac{1}{C_{\varsigma} C_{\eta}}\left[\frac{\partial}{\partial \varsigma}\left(C_{\eta} H u u\right)+\frac{\partial}{\partial \eta}\left(C_{\varsigma} H v u\right)+H v u \frac{\partial C_{\varsigma}}{\partial \eta}-H v^{2} \frac{\partial C_{\eta}}{\partial \varsigma}\right]+\frac{g u \sqrt{u^{2}+v^{2}}}{C^{2}} \\
& +\frac{g H}{C_{\varsigma}} \frac{\partial h}{\partial \varsigma}-f v H=\frac{1}{C_{\varsigma} C_{\eta}}\left[\frac{\partial}{\partial \varsigma}\left(C_{\eta} H \sigma_{\varsigma \varsigma}\right)+\frac{\partial}{\partial \eta}\left(C_{\varsigma} H \sigma_{\varsigma \eta}\right)+H \sigma_{\varsigma \eta} \frac{\partial C_{\varsigma}}{\partial \eta}-H \sigma_{\eta \eta} \frac{\partial C_{\eta}}{\partial \varsigma}\right]
\end{aligned}
$$




$$
\begin{aligned}
& \frac{\partial(H v)}{\partial t}+\frac{1}{C_{\varsigma} C_{\eta}}\left[\frac{\partial}{\partial \varsigma}\left(C_{\eta} H u v\right)+\frac{\partial}{\partial \eta}\left(C_{\varsigma} H v v\right)+H v u \frac{\partial C_{\eta}}{\partial \varsigma}-H u^{2} \frac{\partial C_{\varsigma}}{\partial \eta}\right]+\frac{g v \sqrt{u^{2}+v^{2}}}{C^{2}} \\
& +\frac{g H}{C_{\eta}} \frac{\partial h}{\partial \eta}+f u H=\frac{1}{C_{\varsigma} C_{\eta}}\left[\frac{\partial}{\partial \varsigma}\left(C_{\eta} H \sigma_{\varsigma \eta}\right)+\frac{\partial}{\partial \eta}\left(C_{\varsigma} H \sigma_{\eta \eta}\right)+H \sigma_{\varsigma \eta} \frac{\partial C_{\eta}}{\partial \varsigma}-H \sigma_{\varsigma \varsigma} \frac{\partial C_{\varsigma}}{\partial \eta}\right]
\end{aligned}
$$

In the above formula, $u, v$ refers to $\zeta, \eta$ velocity component, $h$ refers to Water level, $H$ refers to Water depth, $g$ refers to Acceleration of gravity, $f$ refers to Coriolis force coefficient, $C_{\varsigma}, C_{\eta}$ as follows(Eq.4 5):

$$
\begin{aligned}
& C_{\zeta}=\sqrt{x_{\varsigma}^{2}+y_{\zeta}^{2}} \\
& C_{\eta}=\sqrt{x_{\eta}^{2}+y_{\eta}^{2}}
\end{aligned}
$$

$\sigma_{\varsigma \zeta}, \sigma_{\eta \eta}, \sigma_{\varsigma \eta}, \sigma_{\eta \varsigma}$ refers to stress, $v_{t}=a u_{*} \Delta s, a$ refers to coefficient, $u *$ refers to the friction velocity, $\triangle s$ refers to space step.

The numerical calculation method and the grid type. The basic equations using ADI method, ADI method (alternating direction method) is proposed by Peaceman, Rachford and Douglas in 1955, its main technical route is: let $\triangle t, \triangle x, \triangle y$ were the time step and $x, y$ direction space step, step $n, i, j$ number of layers and are too $x, y$; in $x \sim y$ plane using staggered grid, and to the various variables $(z, u, v, h)$ calculations; in time by using the $\triangle t$ is divided into 2.5 steps, calculated by implicit, explicit alternating implicit, explicit, in $n \triangle t \sim(n+1 / 2) \triangle t$ half step with the momentum equation implicit discrete continuity equation and the $x$ direction, and with the pursuit method $z$ and $u$ layers on the $(n+1 / 2) \triangle t$, the momentum equations in the $y$ direction with the explicit discrete, and the $v$ layer on the $(n+1 / 2) \triangle t$, and then in the $(n+1 / 2) \triangle t \sim(n+1) \triangle t$ for the discrete continuity equation and momentum equation in the $y$ direction with implicit $\triangle t$ half step, and with the pursuit method $z$ and $v$ layers on the $(n+1) \triangle t$, the momentum equations in the $x$ direction with the explicit discrete, and obtain $u$ layer on the $(n+1) \triangle t$. The grid pattern as shown in Figure.1.

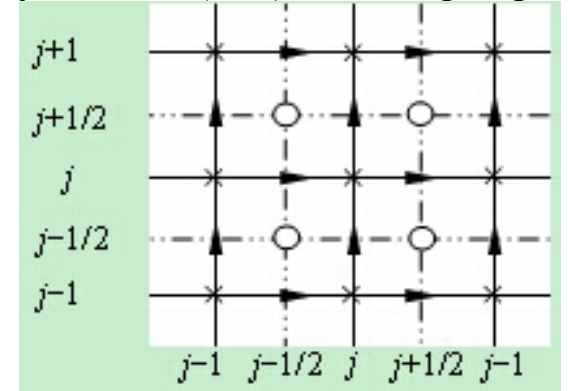

Figure 1. A Two-Dimensional Grid Type Diagram

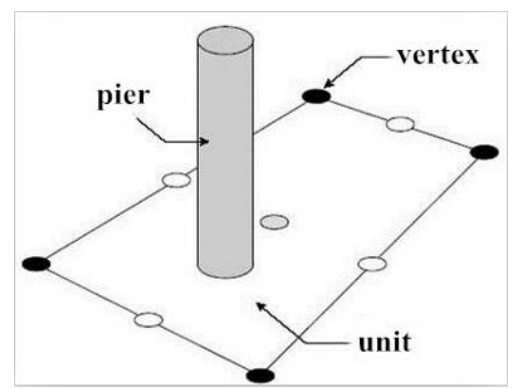

Figure 2. Pier Sketch Map

\section{Based on the Analysis of Flood Control in the Village Station}

Generalization of river engineering. The calculation range of river engineering mainly includes: dike, dangerous section, control project, embankment and production dikes. The calculation of the project was generalized, based on terrain and topography, the project is to improve the grid elevation to the corresponding elevation.

Generalization of River Bridge. Simulation of River Bridge on the river water effect, reasonable generalization pier is the key procedure, if generalization is reasonable, the calculation results can accurately reflect the actual situation. The calculation range of River Bridge equivalent processing. The pier is generalized as follows: the pier where the unit bed roughness is divided into two parts: one is the basic roughness, i.e. no pier bed roughness $n$; two is the equivalent roughness, equivalent 
roughness effects on flow form pier drag and drop rate. Drag the pier on flow will affect the average allocation to the pier where the unit. The basic unit of roughness set obtained by non engineering conditions, equivalent roughness using NANKER Institute of pile group resistance research:

$$
n_{t}=\left(\frac{A^{\prime}}{2} \cdot C_{D} \cdot H^{1 / 3} \cdot \frac{1}{g n^{2} \Delta x \Delta y}+1\right)^{1 / 2} \cdot n \cdot \frac{A_{p}}{A_{e}}
$$

Formula: $n_{t}$ refers to roughness; $n$ refers to bed roughness; $H$ refers to depth; $A^{\prime}$ refers to single column in the projection area perpendicular to the flow direction; $C_{D}$ refers to drag coefficient, single pile or pile group $C_{D}=C_{d} K_{d} N_{d} ; C_{d}$ refers to drag coefficient, $C_{d}=2.0 K_{d}$ : single pile; pile group coefficient, $K_{d}=0.3 \sim 1.0 ; N_{d}$ refers to the number of piles; $\Delta x \Delta y$ refers to pile plane size, $A_{p}$ refers to pier in the projection area of the plane of the element, $A_{e}$ refers to the unit area. Schematic diagram of normalized pier as shown in Figure.2.

Calculation and boundary conditions. The range of the regulation project is from $83+350 \sim 102+800$ of the Yellow River dike, the upstream of the 2D hydrodynamic model was located at $38+000$ while the downstream was located at $120+000$. the length of the model is about $90 \mathrm{~km}$, the simulation domain is discredited using orthogonal curvilinear grid is arranged, $82 \times 662$ (rows and columns), the average mesh size is about $100 \times 110 \mathrm{~m}$. the computing grid layout of the $2 \mathrm{D}$ hydrodynamic model has been displayed in Figure.3.

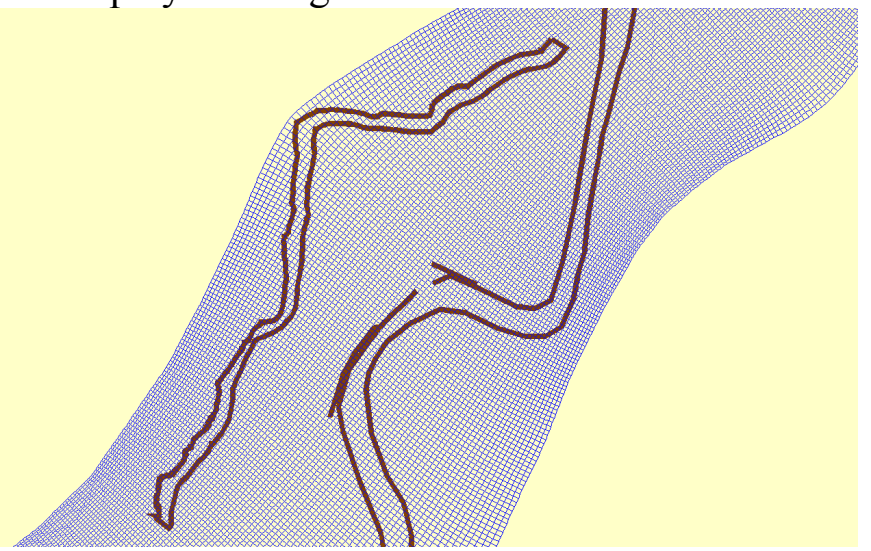

Figure 3: the Computing Grid Layout

The hydrological calculation conditions and conditions. The hydraulic conditions of 2D model should include the discharge of the cross section at the upstream boundary and the water level of the cross section at the downstream boundary. The hydraulic conditions are listed in the table 1 .

Table 1 The hydrological calculation condition

\begin{tabular}{|c|c|c|}
\hline $\begin{array}{c}\text { The upstream condtions } \\
(1 \text { eft dike 38+000) }\end{array}$ & discharge $\left(\mathrm{m}^{3} / \mathrm{s}\right)$ & 20000 \\
\hline $\begin{array}{c}\text { The downstream condtions } \\
(1 \mathrm{eft} \text { dike } 128+800)\end{array}$ & water level $(\mathrm{m})$ & 55.64 \\
\hline
\end{tabular}

\section{The calculation results}

Case 1: current condition means that the dike river has not filled.

Case 2: the elevation of the dike soil $1 \mathrm{~m}$ higher than the average elevation of the beach,

Case 3: the elevation of the dike soil equals the water level with the $4000 \mathrm{~m}^{3} / \mathrm{s} \mathrm{discharge,}$

Case 4: the elevation of the dike soil equals the water level with the $12370 \mathrm{~m}^{3} / \mathrm{s}$ discharge.

By comprising the flow velocities of the case 2,3,4 with the case1, we can obtain the reasonable filling scheme.

The calculation results. According to the results of the 2D model, the change of the average flow velocity in the dike river has been listed in table 2. 
Table 2 the average flow velocity in the dike river after filling

\begin{tabular}{|c|c|c|c|c|}
\hline \multirow{2}{*}{$\begin{array}{c}\text { the range of the } \\
\text { model }\end{array}$} & \multicolumn{4}{|c|}{ the average flow velocity in the dike river $(\mathrm{m} / \mathrm{s})$} \\
\cline { 2 - 5 } & case 1 & case 2 & case 3 & case 4 \\
\hline $\begin{array}{c}83+350 \sim 102+80 \\
0\end{array}$ & 1.10 & 0.88 & 0.38 & 0.20 \\
\hline
\end{tabular}

From table 2, we can know the higher the height of the dike soil is higher, the average flow velocity in the dike river is slower. The result of the case 4 is the best, but case 4 will cost the most investment in these 4 cases. Through comprehensive analyzing, we recommend the case 2 which means that the height of the dike soil $1 \mathrm{~m}$ higher than average elevation of the beach.

\section{Conclusions}

The 2-D hydrodynamic mathematical model was used in the secondary suspended river regulation project design, the change of flow velocity in the dike river can be quantified in different dike river filling solutions to display the influence of height of the dike soil. Using the 2-D hydrodynamic mathematical model can improve the efficiency and the quality of the design working and improve the design quality, also provide a valuable reference for other similar project experience. With the development of the project, we believe the $2 \mathrm{D}$ hydrodynamic mathematical model will play more important effect.

\section{References}

[1] Deguan Wang. Computational hydraulics theory and application[M]. Beijing: Science Press, 2011.8, (in Chinese),

[2]Li Wei: Handbook of Hydraulic Calculations [M]. Beijing: China Water \& Power Press, 2006 (in Chinese). 